\title{
The ELM Model to Estimate Energy Demand of China
}

\author{
Shuai Wei ${ }^{1, a}$ \\ ${ }^{1}$ Mineral Resource Strategy and Policy Research Center of China University of Geosciences, \\ School of Economics and Management, China University of Geosciences, \\ Lumo Rd., Wuhan (430074), China \\ aweishuai1991@foxmail.com
}

\section{Keywords: Energy Demand; Projection; ELM; Scenario Analysis}

Abstract: Energy demand is closely bound up with the problem of global climate change, environmental pollution and carbon emissions, which is always a hot issue that the world is paying attention to, especially the energy demand scale is the focus of social attention. Therefore, it is highly urgent to forecast the energy demand accurately. Firstly, this paper summarizes six main factors of energy demand; Then, constructs the prediction model based on ELM, trains and tests the model based on historical sample data of energy demand and its factors; Finally, forecasts evolution rule of China's energy demand as its factors from 2014 to 2035 through setting three scenarios. Conclusions show that: 1) The error rate of ELM model in training phase and testing phase is only $1.35 \%$ and $0.18 \%$ respectively, and the prediction results of energy demand in this paper with the method of ELM model is relatively close to the predicted results of EIA and BP; 2) China's energy demand in future will present a gradual increment trend, which will reach 5.29, 6.61 and 6.92 billion tons of standard coal respectively in 2020, 2030 and 2035 under the baseline scenario, and the energy supply system and carbon emissions system in future will still be under grater pressure; 3) China's energy demand will still experience a rigid growth stage with a high speed, but the growth rate of energy demand will tend to slow down gradually.

\section{Introduction}

With the urbanization and industrialization process accelerating consistently, China's energy demand will increase rapidly. According to "BP Energy Outlook 2035"[1], China will be largest energy importer of the world over Europe in 2035, energy demand will increase by 60 percent compared to 2013 , contributing the 36 percent of the world energy increment. Energy issue is not only related to the healthy development of the national economy, also bound up with the problem of global climate change, environmental pollution and carbon emissions, which is the global and strategic problem that China's sustainable development faces. How to forecast energy demand in future accurately shows important practical significance for China's long-term strategic planning and sustainable development of the national economy.

In recent years, scholars have made a lot of studies on energy demand. In the course of research, some common prediction methods have been obtained, such as sectoral projection ${ }^{[1,2]}$, input-output model $^{[3]}$, elastic coefficient method ${ }^{[4]}$, bottom-up model ${ }^{[5,6]}$, econometric model ${ }^{[7]}$, decomposition model $^{[8,9]}$, system dynamics ${ }^{[10]}$, gray prediction ${ }^{[11,12]}$ and other traditional methods ${ }^{[13]}$. The above-mentioned traditional prediction methods have made a certain contribution to the long strategic planning of energy. However, energy demand system is a complex and highly nonlinear system, which has many interactive affecting factors, resulting in the increase in the complexity and difficulty of the energy demand prediction. In addition, the traditional prediction method has difficulty accurately describing the nonlinear relationship in energy demand system and achieving high prediction precision. Therefore, it is necessary to pursue more scientific and effective methods to forecast energy demand. The ELM, by contrast, is gradually favored by scholars for its advantages, such as faster learning speed and better generalization capability, which has been maturely used in the field of classification, pattern recognition, feature learning and time series prediction at present. Shrivastava et al. (2014) forecasted the short term price of electricity markets based on the method of Hybrid Wavelet-ELM ${ }^{[14]}$; Dash et al. (2014) adopted the self-adaptive differential harmony search 
based optimized ELM to forecast financial time series ${ }^{[15]}$.

From published literature, ELM model has not yet been applied into the field of energy demand prediction. Therefore, this paper constructs ELM model to forecast the development rule of China's energy demand as its influence factors through setting three scenarios.

\section{Methodology}

Extreme Learning Machine (ELM) is a fast learning algorithm of feedforward neural network proposed by Huang in $2004^{[16]}$. It randomly generates the connection weights between input layer and hidden layer and the threshold of hidden layer neurons without adjustment in the training process, obtains the only optimal solution by setting the number of hidden layer neurons.

As for any $N$ independent training samples $\left\{\left(x_{i}, t_{i}\right), i=1,2, \mathrm{~L}, N\right\}$, where, $x_{i}=\left[x_{i 1}, x_{i 2}, \mathrm{~L}, x_{i n}\right]^{T} \in R$ is the input value of sample, $t_{i}=\left[t_{i 1}, t_{i 2}, \mathrm{~L}, t_{i m}\right]^{T} \in R$ is the expected output value of the sample. Given the activation function of the network is $g(x)$, the threshold of the hidden layer neurons is $b_{i}$, the output of the network could be got by the Fig.1:

$$
y_{j}=\sum_{i=1}^{l} \beta_{i} g_{i}\left(x_{j}\right)=\sum_{i=1}^{l} \beta_{i} g_{i}\left(w_{i} x_{j}+b_{i}\right)
$$

where, $j=1,2, \mathrm{~L}, N, w_{i}=\left[w_{i 1}, w_{i 2}, \mathrm{~L}, w_{i n}\right]$ is the connection weight between the input layer neurons and the $i$ th neuron of the hidden layer, $\beta_{i}=\left[\beta_{i 1}, \beta_{i 2}, \mathrm{~L}, \beta_{i m}\right]$ is the connection weight between the $i$ th neuron of the hidden layer and the output layer neurons.

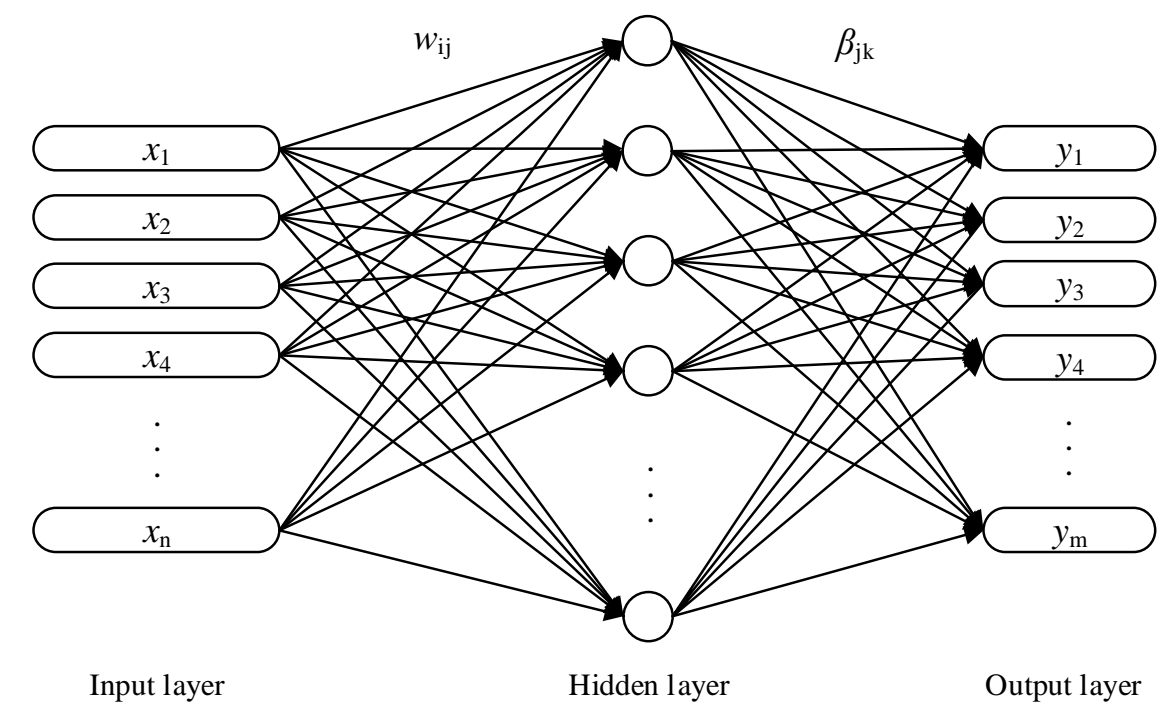

Fig.1. Typical single-hidden layer feedforward neural network structure

Formula (1) could be converted into:

$Y=H \beta$

where, $Y$ is the output of network, and $H$ is the output matrix of the hidden layer of the network:

$$
\begin{aligned}
& H\left(w_{1}, w_{2}, \mathrm{~L}, w_{l} ; b_{1}, b_{2}, \mathrm{~L}, b_{l}, x_{1}, x_{x}, \mathrm{~L}, x_{n}\right) \\
& =\left[\begin{array}{cccc}
g\left(w_{1} \cdot x_{1}+b_{1}\right) & g\left(w_{2} \cdot x_{1}+b_{2}\right) & \mathrm{L} & g\left(w_{l} \cdot x_{1}+b_{l}\right) \\
g\left(w_{1} \cdot x_{2}+b_{1}\right) & g\left(w_{2} \cdot x_{2}+b_{2}\right) & \mathrm{L} & g\left(w_{l} \cdot x_{2}+b_{l}\right) \\
\mathrm{M} & \mathrm{M} & \mathrm{L} & \mathrm{M} \\
g\left(w_{1} \cdot x_{n}+b_{1}\right) & g\left(w_{2} \cdot x_{n}+b_{2}\right) & \mathrm{L} & g\left(w_{l} \cdot x_{k}+b_{l}\right)
\end{array}\right]_{n \times l}
\end{aligned}
$$


On the basis of previous studies, Huang et al. proved that $\left.{ }^{[16,17]}: 1\right)$ For any $N$ independent training samples $\left(x_{i}, t_{i}\right)$, where, $x_{i}=\left[x_{i 1}, x_{i 2}, \mathrm{~L}, x_{i n}\right]^{T} \in R, t_{i}=\left[t_{i 1}, t_{i 2}, \mathrm{~L}, t_{i m}\right]^{T} \in R$, if the node of hidden layer has the same number with the training data, as with any $w_{i} \in R$ and $b_{i} \in R$, the network can approximate training samples with zero error; 2) For any $n$ independent training samples $\left(x_{i}, t_{i}\right)$, where, $x_{i}=\left[x_{i 1}, x_{i 2}, \mathrm{~L}, x_{i n}\right]^{T} \in R, t_{i}=\left[t_{i 1}, t_{i 2}, \mathrm{~L}, t_{i m}\right]^{T} \in R$, if the number of hidden layer node is less than the sample number of training data, in the circumstance that there are any small error $\varepsilon$ and the activation function $g(x)$ that is infinitely differentiable in arbitrary interval, there is always a SLFN containing $k(k \leq N)$ hidden layer neurons, as with any $w_{i} \in R$ and $b_{i} \in R$, there is $\|H \beta-Y\|<\varepsilon$.

Therefore, when the activation function $g(x)$ is infinitely differentiable, the parameters of SLFN do not need to be adjusted, and $w$ and $b$ could be selected randomly before training and remain unchanged in the process of training. And the connection weight $\beta$ between the hidden layer and the output layer can be obtained by solving the least square solution of the following equation:

$$
\underset{\beta}{\operatorname{Min}\|H \beta-Y\|}
$$

Its solution is:

$$
\beta=H^{+} T
$$

Where, $H^{+}$is Moore-Penrose generalized inverses of matrices of the output matrix $H$ in the hidden layer.

\section{Empirical study of energy demand prediction}

This paper selects six indicators based on the literatures available, including GDP, energy processing and conversion efficiency, total population, proportion of coal in energy consumption, urbanization rate and secondary industry proportion, as the main factors affecting energy demand.

Data source and pretreatment

The time interval of the sample data is selected from 1980 to 2013, the prediction interval of energy demand is selected from 2014 to 2035, the sample data mainly comes from the "China Statistical Yearbook 2015". This paper conducts the pretreatment for the raw data with the method of normalization to make the data mapped to $[0,1]$, the formula of normalization is as follows:

$$
x_{i j}^{*}=\frac{x_{i j}-x_{\text {Min }}}{x_{M a x}-x_{M i n}}
$$

In formula (6), $x_{i j}$ is the original data, $x_{i j}^{*}$ is the normalized data, $x_{M a x}$ and $x_{\text {Min }}$ are the maximum and minimum of the data respectively.

Training and testing of ELM model

This paper selects the six influence factors of energy demand as the input variables of ELM model, selects energy demand as the output variables of ELM model, selects the sigmoid function as the activation functions of ELM model, trains ELM model based on the sample data from 1980 to 2011 and tests ELM model based on the sample data from 2012 to 2013 to examine the prediction effect of ELM model, determine 15 as hidden layer nodes by trial and error, then train and test ELM model, the process above is realized in the Matlab2015b, the actual data and the simulated data of ELM model from 1980 to 2013 one time are shown in Fig.2. 


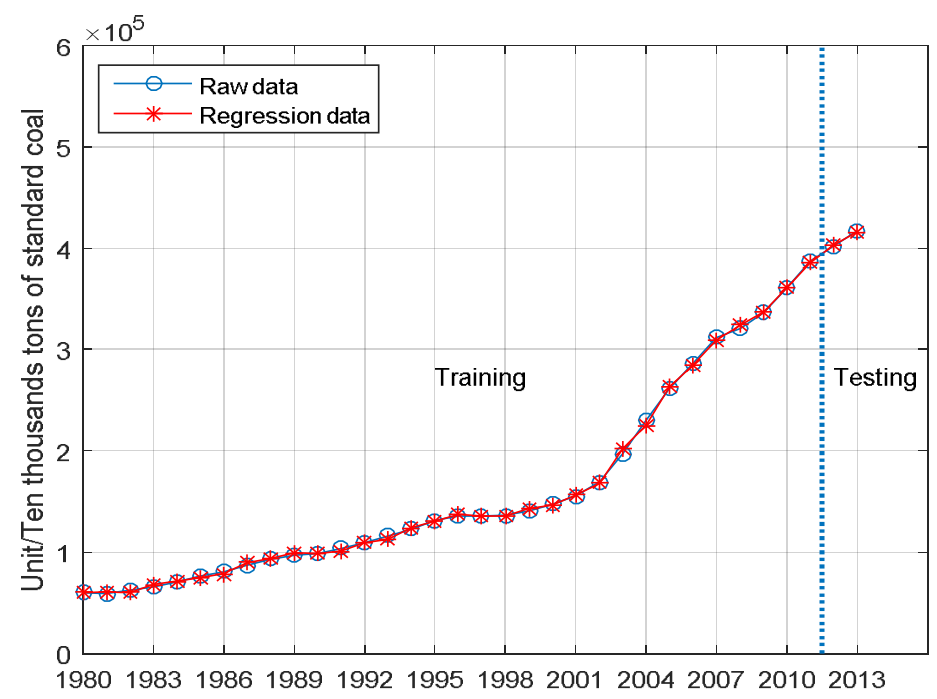

Fig.2. Prediction results of ELM model

To show the prediction accuracy of ELM model, this paper adopts the mean absolute percentage error (MAPE) to evaluate the error rate of model, the formula is as follows:

$$
\text { MAPE }=\frac{\left|y_{i}-\hat{y}_{i}\right|}{y_{i}}
$$

where, $y_{i}$ and $\hat{y}_{i}$ are the actual values and predicted values of energy demand respectively. It can be seen that the MAPE of ELM model in training phase and testing phase are $1.35 \%$ and $0.18 \%$ respectively, and the maximum error of a single year is $3.9 \%$, which shows higher prediction precision.

China's energy demand prediction based on scenario analysis

\section{Scenario scheme setting}

In order to better illustrate the evolution law of energy demand as different indicators, this paper firstly sets three different scenarios, explores the variation of six factors under different scenarios, and then forecast China's energy demand from 2014 to 2035. This paper divides the time interval of scenario analysis into three phases: 2014-2020, 2021-2030 and 2031-2035.

Scenario scheme 1: Baseline scenario. In this scenario, energy demand develops under the existing potential, each indicator has a general operation condition, it specifically demonstrates that: The annual growth rate of GDP will slow down gradually; Energy processing and conversion efficiency will continue to progress; the population will keep a low speed; Energy consumption structure will continue to be adjusted; The urbanization level will advance steadily; Industrial structure will be continuously optimized.

1) GDP. One of the core objectives of the 13th Five-Year Plan is that GDP in 2020 will be is set to double compared with 2010, average growth rate of GDP in the first three years of the 12th Five-Year Plan (2011-2013) is 8.3\%, on the basis of this, to ensure that GDP in 2020 is double compared with 2010, the minimum bottom of GDP growth rate from 2014 to 2020 is $6.7 \%$. In recent years, economic growth has entered the shift period, the growth rate of GDP has started to take shape. Therefore, under the baseline scenario, this paper sets the growth rate of GDP in 2014-2020, 2021-2030, and $2031-2035$ as $7 \%, 6 \%$ and $5 \%$ respectively.

2) Energy processing and conversion efficiency. To improve energy efficiency is the core content of the 13th Five-Year energy planning. In fact, it is relatively easy to improve energy efficiency in low efficiency, the more difficult improving energy efficiency, the higher energy efficiency ${ }^{[18]}$. Since the 11th Five-Year Plan, the annual growth rate of energy processing and conversion efficiency is $0.32 \%$. Therefore, under the baseline scenario, this paper sets the annual growth rate of energy processing 
and conversion efficiency in $2014-2020,2021-2030$ and $2031-2035$ as $0.32 \%, 0.26 \%$ and $0.2 \%$ respectively.

3) Total population. At present, China's population has entered the low speed growth stage. According to China's population prediction of "world population prospects 2015 "[19] by the United Nations, China's population will respectively reach 1.38, 1.42, 1.42 billion people respectively in 2015, 2028 and 2030, and the peak of population will appear in 2028, and then it will start to decline slowly. In view of this, under the baseline scenario, this paper sets the annual growth rate in 2014-2020 as 0.3\%, then calculate the annual growth rate of population in 2021-2030, namely $0.19 \%$, and set the annual growth rate of population in 2031-2035 as $-0.1 \%$.

4) The proportion of coal in energy consumption. During the 13th Five-Year Plan, reducing the proportion of coal in energy consumption has been the key point. According to "The Strategic Action Plan for Energy Development (2014-2020)",[20], the proportion of coal in energy consumption will be controlled less than $62 \%$ in 2020 . Accordingly, calculate the annual growth rate of the proportion of coal in energy consumption in 2014-2020, namely $-1.2 \%$, and set the annual growth rate of 2021-2030 and 2031-2035 as $-1 \%$ and $-0.8 \%$ respectively.

5) Urbanization rate. According to "The National New Urbanization Plan (2014-2020)" [21], China's urbanization rate will reach $60.34 \%$ in 2020 , on the basis of this, it is calculated that the baseline of urbanization rate in 2014-2020 is $1.6 \%$. Therefore, under the baseline scenario, this paper sets the annual growth rate of urbanization rate in 2014-2020, 2021-2030 and 2031-2035 as 1.6\%, $1.3 \%$ and $1 \%$ respectively.

6) Secondary industry proportion. In recent years, a series of industrial policies have been imposed to adjust and upgrade the industrial structure from the national level. Chinese Academy of Social Sciences predicted that the proportion of secondary industry in the tertiary industry would drop to $41 \%$ in 2020 . Then, calculate the annual growth rate of secondary industry in 2014-2020, namely $-0.9 \%$, and set annual growth rate in $2021-2030$ and $2031-2035$ as $-0.75 \%$ and $-0.6 \%$ respectively.

Scenario scheme 2: High growth scenario. Compared with the baseline scenario, each indicator in high growth scenario maintains high speed growth. Refer to the baseline scenario, this paper sets annual growth rate of GDP in 2014-2020, 2021-2030 and 2031-2035 as $8.5 \%, 7 \%$ and $5.5 \%$ respectively; Set the annual growth rate of energy processing and conversion efficiency as $0.4 \%$, $0.32 \%$ and $0.24 \%$ respectively. Set the annual growth rate of total population as $0.4 \%, 0.25 \%$ and $0.1 \%$ respectively; Set the annual growth rate of proportion of coal in energy consumption as $-0.9 \%$, $-0.75 \%$ and $-0.6 \%$ respectively; Set the annual growth rate of urbanization as $2 \%, 1.6 \%$ and $1.2 \%$ respectively; Set the annual growth rate of secondary industry proportion as $-0.6 \%,-0.5 \%$ and $-0.4 \%$ respectively.

Scenario scheme 3: Low growth scenario. Compared with the baseline scenario, energy demand under low growth scenario is restrained to some extent. Refer to the baseline scenario, this paper sets annual growth rate of GDP in 2014-2020, 2021-2030 and 2031-2035 as 6\%, 5\% and 4\% respectively; Set the annual growth rate of energy processing and conversion efficiency as $0.24 \%, 0.2 \%$ and $0.16 \%$ respectively. Set the annual growth rate of total population as $0.2 \%, 0.1 \%$ and $-0.2 \%$ respectively; Set the annual growth rate of proportion of coal in energy consumption as $-1.5 \%,-1.2 \%$ and $-0.9 \%$ respectively; Set the annual growth rate of urbanization as $1.2 \%, 1 \%$ and $0.8 \%$ respectively; Set the annual growth rate of secondary industry proportion as $-1.5 \%,-1.2 \%$ and $-0.9 \%$ respectively.

The variations of influencing factors under different scenarios are shown in Table 1. 
Table 1 Annual growth rates of factors in different stages[\%]

\begin{tabular}{|c|c|c|c|c|c|c|c|}
\hline $\begin{array}{l}\text { Scenario } \\
\text { scheme }\end{array}$ & Year & GDP & $\begin{array}{c}\text { Energy } \\
\text { processing and } \\
\text { conversion } \\
\text { efficiency }\end{array}$ & $\begin{array}{c}\text { Total } \\
\text { population }\end{array}$ & $\begin{array}{l}\text { The proportion of } \\
\text { coal in energy } \\
\text { consumption }\end{array}$ & $\begin{array}{l}\text { Urbanization } \\
\text { rate }\end{array}$ & $\begin{array}{l}\text { Secondary } \\
\text { industry } \\
\text { proportion }\end{array}$ \\
\hline \multirow{3}{*}{$\begin{array}{l}\text { Baseline } \\
\text { scenario }\end{array}$} & $2014-2020$ & 7 & 0.32 & 0.3 & -1.2 & 1.6 & -0.9 \\
\hline & $2021-2030$ & 6 & 0.26 & 0.19 & -1.0 & 1.3 & -0.75 \\
\hline & $2031-2035$ & 5 & 0.20 & -0.1 & -0.8 & 1.0 & -0.6 \\
\hline \multirow{3}{*}{$\begin{array}{l}\text { High } \\
\text { growth } \\
\text { scenario }\end{array}$} & $2014-2020$ & 8.5 & 0.4 & 0.4 & -0.9 & 2.0 & -0.6 \\
\hline & 2021-2030 & 7 & 0.32 & 0.25 & -0.75 & 1.6 & -0.5 \\
\hline & $2031-2035$ & 5.5 & 0.24 & 0.1 & -0.6 & 1.2 & -0.4 \\
\hline \multirow{3}{*}{$\begin{array}{c}\text { Low } \\
\text { growth } \\
\text { scenario }\end{array}$} & 2014-2020 & 6 & 0.24 & 0.2 & -1.5 & 1.2 & -1.5 \\
\hline & $2021-2030$ & 5 & 0.2 & 0.1 & -1.2 & 1.0 & -1.2 \\
\hline & $2031-2035$ & 4 & 0.16 & -0.2 & -0.9 & 0.8 & -0.9 \\
\hline
\end{tabular}

China's energy demand prediction under different scenarios

Based on the growth rates in different phases shown in Table 1, calculate the values of influencing factors over the years under three scenarios, load the values of influencing factors under different scenarios into ELM model trained well, obtain prediction results of China's energy demand as shown in Fig. 3.

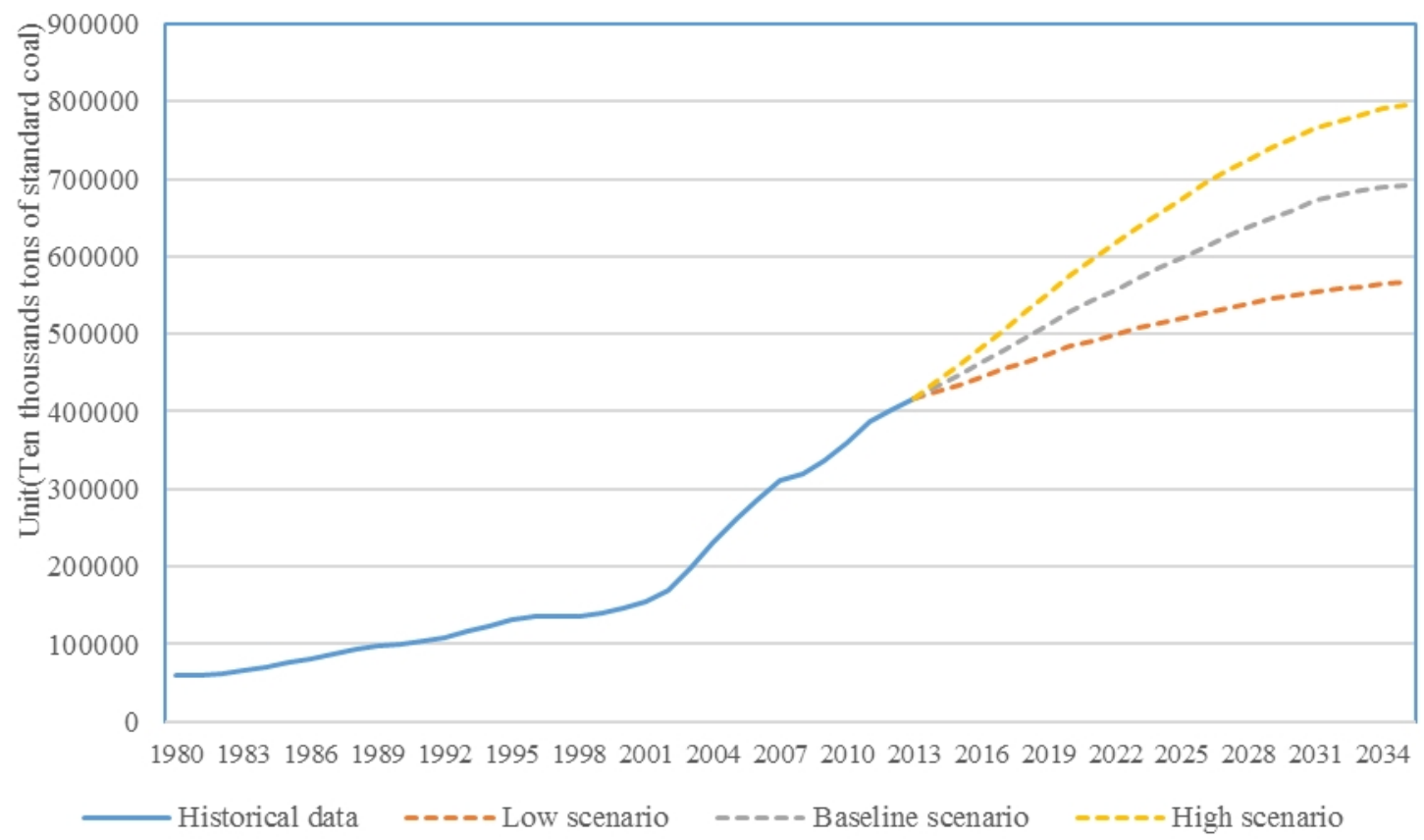

Fig.3. Prediction results of China's energy demand in different scenario schemes

It can be seen from the forecast results of China's energy demand: 1) Energy demand in the three critical years and the growth rate of energy demand under three scenarios have a greater difference. 2) It can be seen that improving the energy utilization efficiency, adjusting the energy consumption structure, promoting urbanization steadily and upgrading industrial structure have positive impact on inhibition of energy demand growth by comparing three scenarios.3) Energy demand will show a tendency to increase under three scenarios, and there is no peak before 2035 yet under three scenarios. But the growth rates of energy demand under three scenarios will show the sign of slowing, energy demand will gradually tend to be saturated.

And this paper compares the prediction results of energy demand with $\mathrm{EIA}^{[22]}$ and $\mathrm{BP}^{[23]}$ in three critical years: 2020, 2030 and 2035, the comparison of the prediction results shows that: Under the baseline scenario, the prediction results of China's energy demand are very closed to that of BP, the 
error in 2020, 2030 and 2035 is only $1.1 \%, 3.8 \%$ and $3.7 \%$ respectively; The predicted results of China's energy demand are also relatively closed to that of BP, the error in 2020, 2030 and 2035 is only $7.6 \%, 7.7 \%$ and $9.9 \%$ respectively.

\section{Conclusions}

This paper forecasts China's energy demand in future by setting three scenario based on ELM model, according to the research process and results, the following conclusions are drawn:

(1) This paper forecasts China's energy demand in future based on ELM model, MAPE of training phase and testing phase is only $1.35 \%$ and $0.18 \%$ respectively, indicating that ELM model has higher prediction accuracy and nonlinear approximation ability, which provides a new approach for the energy demand prediction. The comparison of the prediction results of China's energy demand between this paper and EIA and BP shows that the prediction results in this paper are relatively reasonable.

(2) Under the baseline scenario, China's energy demand will reach 5.29, 6.61 and 6.92 billion tons of standard coal in three critical years: 2020, 2030 and 2035, the annual growth rate in three phases: 2014-2020, 2021-2030 and 2031-2035 is 3.5\% and 2.3\% and 0.9\% respectively, since China have not completed the industrialization and urbanization process, China's energy demand will still experience a rigid growth stage with a high speed.

(3) China's energy demand in future will show a tendency to increase under three scenarios and there is no peak under three scenarios before 2035 yet, the energy supply system and carbon emissions system in future will be under grater pressure. It is worth mentioning that the growth rates of energy demand under the three scenarios will slow down gradually, indicating that China's energy demand will gradually enter into a low-speed growth phase, in view of the growth rate of energy demand, the peak of energy demand in China will possible appear soon after 2035.

\section{Acknowledgements}

We would like to acknowledge that this paper was supported by the National Natural Science Foundation, China (Nos. 71301153); the Fundamental Research Funds for the Central Universities, China University of Geosciences (Wuhan) (Nos. CUG140612); the Scientific Research Foundation for the Returned Overseas Chinese Scholars, State Education Ministry; the Center for digital business and intelligent management.

\section{References}

[1] British Petroleum (BP). (2015) 'BP Energy Outlook 2035'.

[2] World Energy Outlook 2015. International Energy Agency, 2015.

[3] Y.X. He, S.L. Zhang, Y.S. Zhao, et al.: submitted to Energy Conversion and Management (2011).

[4] H.A. Amarawickrama and L.C. Hunt : submitted to Energy (2008).

[5] R. Shabbir and S.S. Ahmad : submitted to Energy (2010).

[6] Y. Huang, Y.J. Bor and C.Y. Peng : submitted to Energy Policy (2010).

[7] V.S. Ediger and S. Akar : submitted to Energy Policy (2007).

[8] B.W. Ang and P.W. Lee : submitted to Energy Economics (1996).

[9] J.W. Sun : submitted to Energy (2001).

[10]Z. Tao : submitted to Energy (2010).

[11]D. Akay and M. Atak : submitted to Energy (2007).

[12] Y.S. Lee and L.L. Tong : submitted to Energy Conversion and Management (2012). 
[13]L. Suganthi and A.A. Samuel : submitted to Renewable and Sustainable Energy Reviews (2012).

[14]N.A. Shrivastava and B.K. Panigrahi : submitted to Electrical Power and Energy Systems (2014).

[15]R. Dash, P.K. Dash and R. Bisoi : submitted to Swarm and Evolutionary Computation (2014).

[16] G.B. Huang, Q.Y. Zhu and C.K. Siew : submitted to Neurocomputing (2006).

[17]G.B. Huang, C. Lei, C.K. Siew : submitted to IEEE Transactions on Neural Networks (2006).

[18]Z.J. Jiang and B.Q. Lin : submitted to Energy Policy (2012).

[19] World Population Prospects. The United Nations, 2015.

[20]The Strategic Action Plan for Energy Development. Bei Jing: Office of the State Council, 2014[in Chinese].

[21]The National New Urbanization Plan(2014-2020). Bei Jing: Office of the State Council, 2014[in Chinese].

[22] Information on http: //www.eia.gov.

[23]Information on http://www.bp.com. 